\title{
Physical properties and band structure parameters of solid solutions $\mathrm{Hg}_{1-\mathrm{x}-\mathrm{y}} \mathrm{Mg}_{\mathrm{x}} \mathrm{Mn}_{\mathrm{y}} \mathrm{Te}$
}

\author{
Frasunyak V.M.,. Gorley P.M. and Chupyra S.M. \\ Yuri Fedkovych Chernivtsi National University, 2 Kotsyubynsky St., 58012 \\ Chernivtsi, Ukraine, e-mail: semicon@chnu.cv.ua
}

Received: 23.11.2007

\begin{abstract}
We present the results of mechanical, electrical, optical and magnetic characterizations of quaternary solid solutions $\mathrm{Hg}_{1-x-y} \mathrm{Mg}_{x} \mathrm{Mn}_{y} \mathrm{Te}$ for the temperature range of $77-300 \mathrm{~K}$. It is shown that introduction of $\mathrm{Mn}$ and $\mathrm{Mg}$ atoms into the crystalline lattice of HgTe increases microhardness of the alloy. Optimal thermal treatment procedures in the constituent vapours are determined, enabling to control the concentration of active defects and, consecutively, to obtain the materials with different conductivity types and predictable concentrations of carriers. Functional dependences of the band gap and the intrinsic carrier concentration for $\mathrm{Hg}_{1-x-y} \mathrm{Mg}_{x} \mathrm{Mn}_{y} \mathrm{Te}$ on the composition and temperature are obtained.
\end{abstract}

Keywords: solid solutions, absorption spectra, microhardness, band structure.

PACS: 75.40.Cx, 72.80.Ey, 78.40.Fy

UDC: 535.34

\section{Introduction}

Solid solutions based on mercury telluride attract a significant interest in semiconductor material science. They allow significant band gap variations by governing the material composition. The latter property offers very promising prospects of applications in IR devices, UHF electronics and sensor systems [1-4]. Throughout studies have been performed of the technology of $\mathrm{CdHgTe}$ compound, which has become nowadays one of the basic materials for the optoelectronics of infrared spectral range. Although a proper technological optimization has led to creation of high-quality devices, an important question still remains to be solved. It concerns the improvement of reproducibility and stability of material parameters, since due to weak bonds between its components many properties of the crystals, including the concentration of carriers, the conductivity type and the state of defect subsystem degrade with time. In particular, mercury can form a large number of vacancies or diffuse towards the surface of samples and evaporate. The consequences of these processes include degradation of different parameters of the bulk material, together with instability of the surface and junction characteristics for the semiconductor structures. 
The need in improved stability of crystalline lattice with respect to the problem of defect formation triggers a search for new technological approaches. It is predicted that one of the possible ways for solving the problem may consist in introduction of other elements into $\mathrm{HgTe}$ lattice, in addition to cadmium (or even into positions of cadmium). This should strengthen the chemical bonds in the solid solution, improve their mechanical properties and give rise to higher stability of the operating characteristics of numerous devices based on this material. Moreover, this approach would also result in synthesis of new materials, which could potentially become an alternative to canonical $\mathrm{CdHgTe}$. Among them, one should consider first of all quaternary solid solutions $\mathrm{HgCdZnTe}$ and HgCdMnTe [5].

The functional properties of four-component solutions are much wider than those of the ternary ones, because the material parameters can be more accurately fine-tuned by introduction of appropriate impurities. Using the elements with incomplete $3 \mathrm{~d}$ - or $4 \mathrm{f}$ shells, it is possible to make the impurity contributing to a non-compensated magnetic moment of the material. This leads to some unusual properties, if compare with those of the common diamagnetic materials, thus opening new possibilities for the applications of spintronic devices [6]. However, an increase in the number of components involved in the solid solution causes certain technological difficulties for obtaining homogeneous crystals, complicates determination of their exact composition and, moreover, requires a careful approach to interpretation of the experimental results.

In this work we present the results of complex studies for various properties of new quaternary solutions $\mathrm{Hg}_{1-x-y} \mathrm{Mg}_{x} \mathrm{Mn}_{y} \mathrm{Te}(0<x<0.1$ and $0<y<0.1)$ which, in a longerterm outlook, may become an alternative to the solid solutions $\mathrm{Cd}-\mathrm{Hg}-\mathrm{Te}$, due to better and more stable parameters improving reliability of the corresponding devices.

\section{Samples and investigation methods}

In order to synthesize the desired alloys, we used the following initial components: $P \cdot 10^{-6}$ mercury, magnesium and manganese (with the initial purity 99.998; purified additionally by a three-cycle vacuum distillation), and a band-purified tellurium $\left(p_{77 \mathrm{~K}} \leq 10^{14} \mathrm{~cm}^{-3}\right)$. The charge was prepared by melting the components together in the pumped-out and sealed quartz containers, the inner surface of which was covered by pyrolithic carbon. The melting temperature of the alloy was determined from differential thermal analysis. Monocrystallization of the alloy was performed by the Bridgman method under the temperature gradients about $25-30 \mathrm{~K} / \mathrm{cm}$ at the crystallization edge. The samples for studies of mechanical, electrical, optical and magnetic properties were made of the plates cut from different parts of the ingot transversally to its growth axis. A defect layer formed upon cutting and preliminary coarse polishing was removed by fine polishing with a diamond paste and further etching of the samples in the solution of bromine in dymethilformamide. The composition and homogeneity of the component distribution was inspected while studying the density (by hydrostatic weighting) and magnetic 
susceptibility $\chi$, which was measured with the Faraday method in the temperature range of 77-300 K under the magnetic fields 0.05-0.90 T.

Microhardness of $\mathrm{Hg}_{1-x-y} \mathrm{Mg}_{x} \mathrm{Mn}_{y}$ Te samples was investigated using a standard Vickers hardness test on PMT-3 equipment under the load of $50 \mathrm{~g}$, corresponding to microhardness plateau for all the samples. The pressure was applied for $5 \mathrm{~s}$ and the values of microhardness obtained were averaged over 10-15 measurements. Galvanomagnetic effects were studied for the both virgin and annealed samples for the temperatures $77-300 \mathrm{~K}$ and the magnetic fields up to $1.2 \mathrm{~T}$. The samples had a standard Hall configuration and the ohmicity of the contacts was verified with the aid of measurements of their current-voltage curves. Finally, the optical properties of the samples were derived using spectrometer IKS-31 in the temperature range of 80-300 K.

\section{Measurement results and their analysis}

The differences in the properties of $\mathrm{HgTe}, \mathrm{MnTe}$ and especially MgTe allow tracking the density changes for the multi-component solid solution as a function of its composition. It has been proved with significant precision that, in the composition range under study, this dependence is linear for the both $\mathrm{Hg}_{1-x} \mathrm{Mg}_{x} \mathrm{Te}$ and $\mathrm{Hg}_{1-y} \mathrm{Mn}_{y} \mathrm{Te}$ compounds. To determine the parameters of the quaternary solid solutions, we have made use of the known parameters for the ternary systems [7], which enables one to obtain the following relation for the density of $\mathrm{Hg}_{1-x-y} \mathrm{Mg}_{x} \mathrm{Mn}_{y} \mathrm{Te}$ :

$$
\rho(x, y)=8.08-4.285 x-3.222 y \text {. }
$$

Eq. (1) has been used to estimate the magnesium content in the samples for the given fraction of manganese obtained from the magnetic susceptibility studies.

Studies of magnetic susceptibility have shown that all of $\mathrm{Hg}_{1-x-y} \mathrm{Mg}_{x} \mathrm{Mn}_{y} \mathrm{Te}$ samples are featured by the increase of paramagnetism under either higher Mn concentration or lower temperatures, which is caused by orientation of non-compensated magnetic moments of $\mathrm{Mn}$ ions in the magnetic field. In the temperature range under test, $\chi_{\mathrm{Mn}}(T)$ obeys well the Curie-Weiss law:

$$
\chi_{M n}(T)=\frac{N_{A}}{3} \frac{g^{2}}{k} \frac{\mu_{B}^{2}}{M} \frac{S(S+1)}{(T-\Theta)} y,
$$

where $g=2$ is the Lande $g$-factor, $\mu_{\mathrm{B}}$ the Bohr magneton, $S=5 / 2$ the spin of Mn ions, $N_{\mathrm{A}}$ the Avogadro's number, $M$ the molecular mass of $\mathrm{Hg}_{1-x-y} \mathrm{Mg}_{x} \mathrm{Mn}_{y} \mathrm{Te}, y$ the contents of manganese ions, $k$ the Boltzman constant and $\Theta$ the paramagnetic Curie-Weiss temperature. The absence of any peculiarities on the $\chi_{\mathrm{Mn}}(T)$ and $\chi_{\mathrm{Mn}}(\mathrm{H})$ curves for the temperature and composition ranges studied by us proves a single-phase nature of the samples on a macroscopic level. The experimental results have been processed using the methodology described in the work [8] and employed to study the temperature dependence of magnetic susceptibility for $\mathrm{Hg}_{1-x-} \mathrm{Mn}_{x} \mathrm{Te}$ and to determine the manganese 
concentration in those samples. The main parameters of the materials obtained by us are presented in Table 1.

Table 1. Parameters of $\mathrm{Hg}_{1-\mathrm{x}-\mathrm{y}} \mathrm{Mg}_{\mathrm{x}} \mathrm{Mn}_{\mathrm{y}} \mathrm{Te}$ samples

\begin{tabular}{|c|c|c|c|c|c|}
\hline$\#$ & $\rho, \mathrm{g} / \mathrm{cm}^{3}$ & $\chi, 10^{-6} \mathrm{~cm}^{3} / \mathrm{g}$ & $|\Theta|, \mathrm{K}$ & $x$ & $y$ \\
\hline 1 & 7.74 & 0.51 & 10 & 0.065 & 0.018 \\
\hline 2 & 7.67 & 1.20 & 18 & 0.071 & 0.034 \\
\hline 3 & 7.57 & 1.92 & 28 & 0.080 & 0.051 \\
\hline 4 & 7.53 & 2.40 & 34 & 0.080 & 0.063 \\
\hline 5 & 7.42 & 3.17 & 44 & 0.092 & 0.082 \\
\hline
\end{tabular}

Replacement of mercury atoms by the other elements in $\mathrm{HgTe}$ lattice modifies the length of R(A-B) bond. It has been supposed that shorter bonds in $\mathrm{Mg}-\mathrm{Te}(2.717 \AA)$ and Mn-Te $(2.746 \AA)$, if compare with those in Cd-Te $(2.806 \AA)$, should stabilize the solid solutions $\mathrm{Hg}_{1-\mathrm{x}-\mathrm{y}} \mathrm{Mg}_{\mathrm{x}} \mathrm{Mn}_{\mathrm{y}} \mathrm{Te}$ and therefore increase the hardness of the material. In its turn, this should decelerate formation and movement of dislocation loops and improve structural perfection of the crystal $[9,10]$.

Fig. 1 presents the dependence of microhardness $H$ on the band gap for the samples $\mathrm{Hg}_{1-\mathrm{x}-\mathrm{y}} \mathrm{Mg}_{\mathrm{x}} \mathrm{Mn}_{\mathrm{y}} \mathrm{Te}$ of different compositions. For the sake of comparison, the same figure also presents the curves of $H\left(E_{\mathrm{g}}\right)$ obtained for $\mathrm{Hg}_{1-x} \mathrm{Cd}_{x} \mathrm{Te}$ [4]. As one can see from Fig. 1, the microhardness of samples increases for higher values of $\mathrm{Mg}$ content, which is characteristic of single-phase solid solutions in their homogeneity region. The microhardness of $\mathrm{Hg}_{1-x} \mathrm{Mg}_{x} \mathrm{Te}$ is larger than that of $\mathrm{Hg}_{1-x} \mathrm{Cd}_{x} \mathrm{Te}$, which has the same band gap, and increases upon introduction of manganese. This can prove strengthening of the crystalline lattice bonds for $\mathrm{Hg}_{1-x-y} \mathrm{Mg}_{x} \mathrm{Mn}_{y} \mathrm{Te}$ and deceleration of movement of dislocations, thus improving the structural perfection of the material and so leading to a better quality of the structures created on this basis.

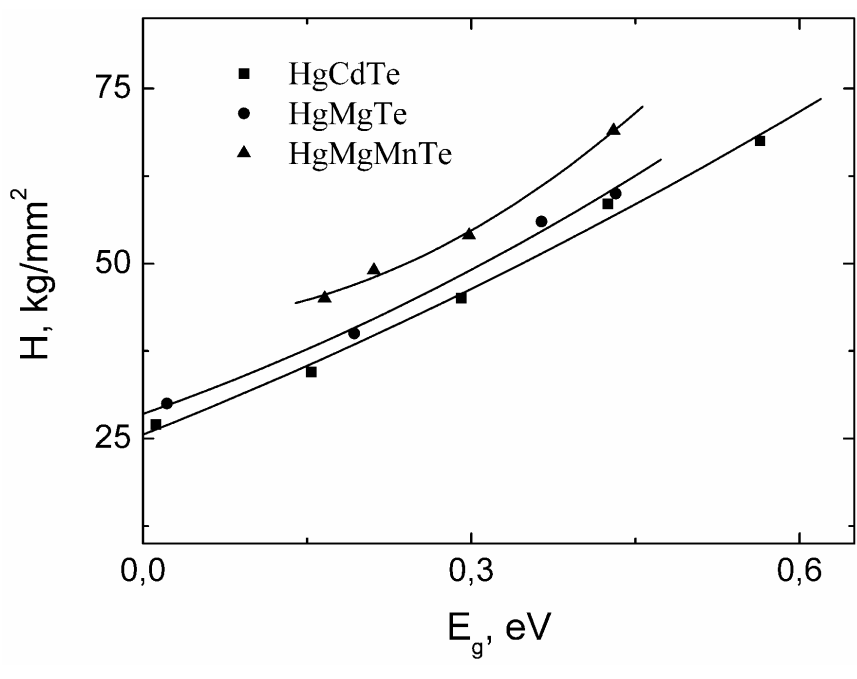

Fig. 1. Microhardness of HgTe-based solid solution crystals as a function of band gap. 
Fig. 2. Temperature dependence of Hall coefficient for the solid $\mathrm{Hg}_{1-x-y} \mathrm{Mg}_{x} \mathrm{Mn}_{y} \mathrm{Te}$ solutions. Curves are numbered according to sample numbers given in Table 1.

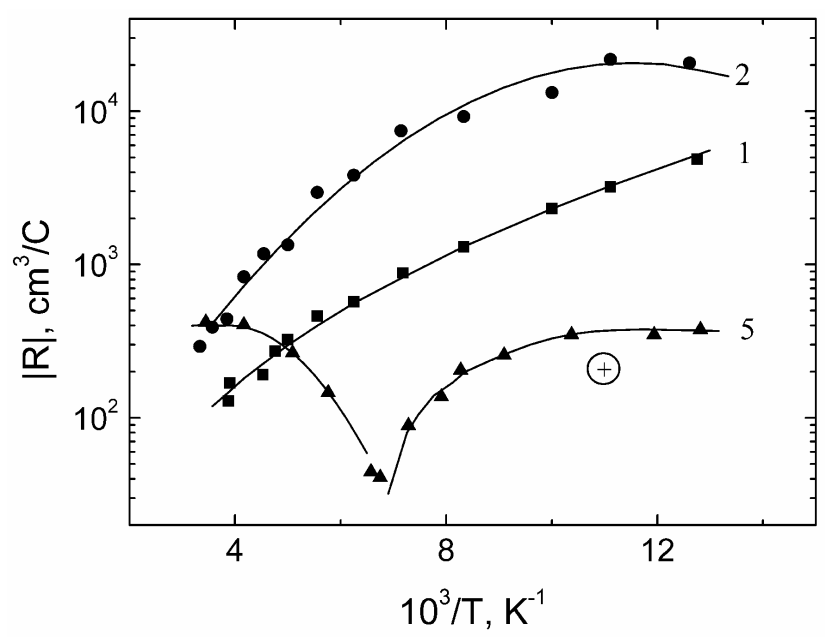

Typical dependence of the Hall coefficient for $\mathrm{Hg}_{1-x-y} \mathrm{Mg}_{x} \mathrm{Mn}_{y} \mathrm{Te}$ crystals versus the reciprocal temperature is shown in Fig. 2. The curves suggest a presence of several types of carriers with different mobilities, which contribute to the transport of charge carriers. For the samples 1 and 2 one can observe the intrinsic conductivity occurring in a wide range. Introduction of manganese leads to the sign inversion for the Hall coefficient (sample 5). To decrease the concentration of defects and obtain materials with a minimal carrier concentration, we have annealed the samples in mercury vapour. We have adjusted the annealing temperature and the elasticity of $\mathrm{Hg}$ vapour, taking into account our previous knowledge deduced from optimization of technology for the ternary systems. With the increase of Mg content (and, in particular, for higher Mn content), we have been forced to increase the annealing time in order to achieve the inversion of conductivity type. The minimal concentration of carriers $\left(n_{77 \mathrm{~K}}=8.3 \cdot 10^{14} \mathrm{~cm}^{-3}\right)$ with the maximum mobility $\left(\mu=2.6 \cdot 10^{5} \mathrm{~cm}^{2} / \mathrm{V} \cdot \mathrm{s}\right)$ is recorded for the solid solution of $\mathrm{Hg}_{0.90} \mathrm{Mg}_{0.04} \mathrm{Mn}_{0.06} \mathrm{Te}$.

Fig. 3. Optical absorption spectra for the $\mathrm{Hg}_{1-x-y} \mathrm{Mg}_{x} \mathrm{Mn}_{y} \mathrm{Te}$ solid solution measured at $T=300 \mathrm{~K}$. Curves are numbered according to sample numbers given in Table 1

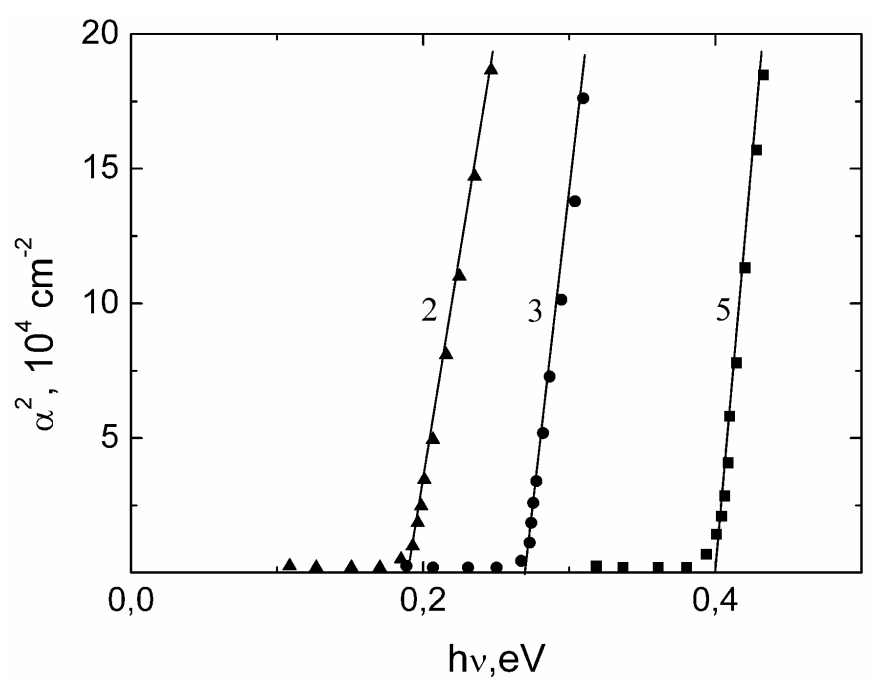

Ukr. J. Phys. Opt. 2008, V9, №1 
In order to determine the band gap $E_{\mathrm{g}}$ of our compounds and find its dependence on the composition, we have studied the fundamental absorption edge for the $\mathrm{Hg}_{1-x-y} \mathrm{Mg}_{x} \mathrm{Mn}_{y} \mathrm{Te}$ crystals. Depending on the composition, the optical transmission coefficient falls into the region of $1-30 \%$. The absorption edge for the materials under test is steep enough to allow estimation of the band gap (Fig. 3). With increasing $\mathrm{Mg}$ and Mn concentrations in the samples, the absorption edge becomes blue-shifted due to a band gap increase. The experimental results have yielded the following expressions describing the band gap and the concentration of intrinsic carriers in the $\mathrm{Hg}_{1-x-y} \mathrm{Mg}_{x} \mathrm{Mn}_{y} \mathrm{Te}$ solid solutions depending on their composition and the temperature:

$$
\begin{gathered}
E_{g}=-0.3+3.918 x+3.649 y+5.036 \cdot 10^{-4} T-(16.503 x+25.5 y) \cdot 10^{-4} T, \\
n_{i}=\left[5.94-6.54 x+2.96 y+2.95 \cdot 10^{-3} T(1+x+y)\right] \cdot 10^{14} E_{g}^{0.75} T^{1.5} \exp \left(-E_{g} / 2 k T\right) .
\end{gathered}
$$

Eqs. (3) and (4) describe successfully the band gap for the solid solutions $\mathrm{Hg}_{1-x-y} \mathrm{Mg}_{x} \mathrm{Mn}_{y} \mathrm{Te}$ and the carrier concentration in the intrinsic conductivity range as functions of the temperature and molar content. However, the agreement of $n_{i}(T)$ with the experiment becomes worse (see Fig. 4) for the lower temperatures, where the system has impurity conduction.

\section{Conclusions}

Therefore, introduction of $\mathrm{Mg}$ and $\mathrm{Mn}$ atoms into the lattice of $\mathrm{HgTe}$, as well as formation of solid solutions $\mathrm{Hg}_{1-x-y} \mathrm{Mg}_{x} \mathrm{Mn}_{y} \mathrm{Te}$, performed with the aid of replacement techniques, improves microhardness of the resulting material, strengthens its chemical bonds and, consequently, influences positively its structure perfection. Using the controlled annealing, we have succeeded in modifying the defect subsystem, right up to changing the conductivity type of the materials under study. We have shown that the absorption edge of $\mathrm{Hg}_{1-x-y} \mathrm{Mg}_{x} \mathrm{Mn}_{y} \mathrm{Te}$ can be shifted towards higher energies by variation

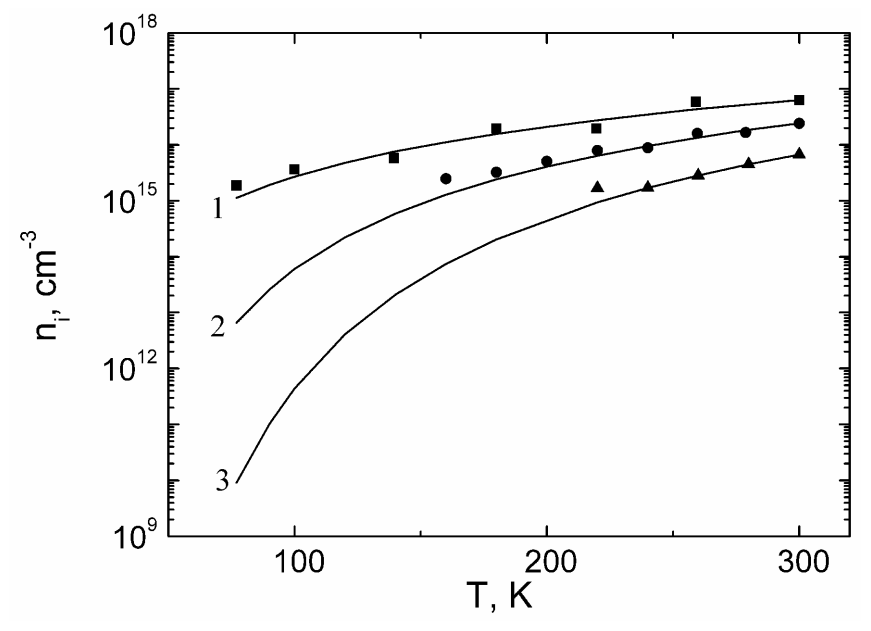

Fig. 4. Temperature dependence of intrinsic carrier concentrations for the $\mathrm{Hg}_{1-x-y} \mathrm{Mg}_{x} \mathrm{Mn}_{y} \mathrm{Te}$ solid solution: experiment (data points) and calculation (curves). Curves are numbered according to sample numbers given in Table 1. 
of the $\mathrm{Mg}$ and $\mathrm{Mn}$ concentrations. The empirical formulae derived by us describe fairly well the dependence of the band gap of the solid solutions and the carrier concentration in the intrinsic conductivity range as functions of the temperature and molar content. The crystals of quaternary solid solutions $\mathrm{Hg}_{1-x-y} \mathrm{Mg}_{x} \mathrm{Mn}_{y} \mathrm{Te}$ suggest wide possibilities for changing their properties based on variations of their composition and temperature. After a proper improvement of their technology, these materials can become a real alternative to the currently used multi-component solid solutions based on the mercury telluride.

\section{References}

1. Baryshev S. N. Properties and applications of narrow-band semiconductors. Kazan: UniPress (2000).

2. Berchenko N. N., Krevs V. E. and Sredin V. G. Semiconductor solid solutions and their applications: Reference tables. Moscow: Voenizdat (1982).

3. Rogalski A, 1991. $\mathrm{Hg}_{1-\mathrm{x}} \mathrm{Mn}_{\mathrm{x}} \mathrm{Te}$ as new infrared detector material. Infrared Phys. 31: 117-166.

4. Rogalski A, 1989. $\mathrm{Hg}_{1-\mathrm{x}} \mathrm{Zn}_{\mathrm{x}} \mathrm{Te}$ as a potential infrared detector material. Progr. Quant. Electr. 13: 299-353.

5. Becla $\mathrm{P}$, 1986. Infrared photovoltaic detectors utilizing $\mathrm{Hg}_{1-\mathrm{x}} \mathrm{Mn}_{\mathrm{x}} \mathrm{Te}$ and $\mathrm{Hg}_{1-\mathrm{x}-\mathrm{y}} \mathrm{Cd}_{\mathrm{x}} \mathrm{Mn}_{\mathrm{y}}$ Te alloys. J. Vac. Sci. and Technol. A4: 2014-2020.

6. Kamilla S K and Basu S, 2002. New semiconductor materials for magnetoelectronics at room temperature. Bull. Mater. Sci. 25: 541-543.

7. Ostapov SE, 2003. Investigation of the main band properties of $\mathrm{HgCdMnZnTe}$. Proc. SPIE 5065: 370-374.

8. Davydov A B, Noskova L M, Ponikarov B B and Ugodnikova L A, 1980. Magnetic susceptibility of $\mathrm{Hg}_{1-\mathrm{x}} \mathrm{Mn}_{\mathrm{x}} \mathrm{Te}$ alloys. Fiz. Tekhn. Poluprov. 14: 1461-1467.

9. Sher A, Chen A B, Spicer W E and Shih C K, 1985. Effects influencing the structural integrity of semiconductors and their alloys. J. Vac. Sci and Technol. A3: 105-111.

10. Bell S L and Sen S, 1985. Crystal growth of $\mathrm{Cd}_{1-\mathrm{x}} \mathrm{Zn}_{\mathrm{x}} \mathrm{Te}$ and its use as a superior substrate for LPE growth of $\mathrm{Hg}_{0,8} \mathrm{Cd}_{0,2}$ Te. J. Vac. Sci and Technol. A3: 112-115. 